\title{
LIQUENES DE LOS PEÑONES DE SAN FRANCISCO (SIERRA NEVADA, SUR DE ESPAÑA)
}

\author{
M. CASARES (*) \& X. LLIMONA (**)
}

\section{RESUMEN}

Aportación a la flora liquénica de la parte esquistosa de Sierra Nevada, en un área abrupta, situada a $2.500 \mathrm{~m}$, próxima al Albergue Universitario. Se da cuenta del hallazgo de 45 especies, entre las cuales: Pilligera degenii, Ramalina obtusiata, Caloplaca epithallina, C. anibon-phoeniceon, Alectoria mintsicula, Parmelia injumata, P'bysconia muscigena, eran poco conocidas o nuevas para España.

\section{SUMMARY}

Contribution to the lichen flora of the Peñones de San Francisco (Sierra Nevada), an abrupt edge of schists, near the University Shelter, at $2.500 \mathrm{~m}$. A blend of 45 mediterranean, orophile and alpine species is reported. Some of them are new to Spain seemingly rare: $P i l$ ligera degenii, Ramalina oblusala, Caloplaca c pilballina, C. ancbon-phoeniceon, Alectoria minuscula. Parmélia infumala, Plysonia muscigena.

Los Peñones de San Francisco comprenden tres elevaciones rocosas, abruptas y apiramidadas, que accidentan la arista que, bajando del Veleta, constituye la divisoria de aguas entre las cuencas de los ríos Monachil y Genil. La variedad de exposiciones, inclinaciones, grietas e incluso cuevas, permite la existencia de líquenes muy diversos, a la vez que abundantes.

La elevación de la localidad, $2.500 \mathrm{~m}$ y su situación en la alta montaña mediterránea, condicionan a la vez una innivación invernal de varios meses, 8 meses con heladas) y una fuerte sequía estival. Lo abrupto y rocoso del relieve y estas duras condiciones climáticas limitan fuertemente la vegetación cormofítica.

Desde el punto de vista litológico, la localidad está situada en la llamada zona de mezcla (Mischungszone), que constituiría los restos del manto superior cristalino de la Sierra Nevada. Está integrado por pizarras bastante enriquecidas en carbonatos. Por ello, aunque la vegetación sea predominantemente silicícola, pueden aparecer en ella especies neutrófilas e incluso calcífilas.

Comunicación presentada al III Simposio Nacional de Botánica Criptogámica. Málaga, 1978.

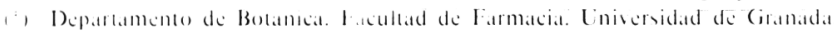

(*) Departamento de Botanica. Facultad de Ciencias. Universidad de Murciat. 
La proximidad del Albergue Universitario y la buena comunicación de la localidad son, en parte, causa de frecuentación y origen de una cierta nitrificación de la zona. Asimismo, explican que se trate de uno de los puntos mejor estudiados de Sierra Nevada. R.G. Werner tuvo ocasión de explorarlos en su ascenso a Sierra Nevada (1933) y publica (Werner, 1936, 1937, 1971, 1979), 17 especies de esta localidad, a saber: Dermatocarpon miniatum, D. rufescens, Lecanora muralis, L. sulphurea, Lecidea carpatbica, L. stigmatea, Parmelia prolixa, P. quercina, Pelligera rufescens, Pertusaria lapieana, Rbizocarpon geographicum, Rinodina atrocinerella, $R$. confragosa, $R$. collectica, Dimelaena oreina, Umbilicaria cylindrica, Xanthoria parielina var. ectanea.

Prieto y Espinosa (1971) citan también algunas especies de la misma localidad: Verrucaria apomelaena, Lecidea rimiseda, Umbilicaria birsula, U. decussala, $U$. subglabra, Acarospora oxytona, parasitada por Rbizocarpon superficiale, Perlusaria inquinata, Lecanora rupicola, L. atrynea var. melacarpa, L. concolor, L. incusa, Rbizoplaia rubina, $R$. melanopthalma, $R$. peltata, Xanthoria elegans, Dimelaena oreina.

Ofrecemos una primera lista de los taxones recogidos por nosotros con algunos comentarios referidos a las especies que nos parecen más interesantes.

\section{LISTA DE ESPECIES}

Dermatocarpon miniatum (L.) Mann. var. imbricatum (Massal.) Vain.

Sobre rocas sombreadas, más o menos húmedas.

Dermatocarpon meiophyllum Vain.

Talo formado por escuámulas de hasta $3 \mathrm{~mm}$, gruesas e imbricadas. Esporas 12’5-15 x 4-6 $\mathrm{nm}$. Sobre rocas protegidas o fisuras de paredes verticales.

Dermatocarpon rufescens (Ach.) Th. Fr.

Sobre suelo en fisuras.

Peltigera degenii Gyel.

Sobre restos vegetales o musgos, en lugares sombreados y húmedos, sobre todo en el interior de grietas rezumantes. Primera cita para la Península Ibérica.

Lecidea atrobrunnea (Ram.) Schaer.

Uno de los líquenes más abundantes y característicos de Sierra Nevada. Se muestra fotófilo, nitrófilo, a menudo ornitocoprófilo.

Lecidea confluens (Web.) Ach.

Talo poco visible. Esporas $7-10 \times 5 \mathrm{~nm}$. Característico de las piedras sueltas, sobre las que resulta pionero.

Lecidea rhaetica Hepp ex Th. Fr.

Talo blanco-amarillento. Epitecio azul verdoso. Esporas $12-15 \times 6-8 \mathrm{~nm}$.

Lecidea tessellata Floerke

Talo fisurado-areolado, grueso, blanco-grisáceo, con hipotalo negro. Epitecio parduzco, hipotecio hialino. Esporas bigutuladas, 6-10 x 5-6 nm. Medula I+ azul.

(nm) micras. 
Rhizocarpon superficiale (Schaer.) Vain.

Como indica Hertel (1970), vive abundante en los extraplomos y techos de los Peñones, muy frecuentemente invadiendo o parasitando Acarospora oxytona.

Rhizocarpon viridiatrum (Wulf.) Koerb.

Talo pequeño. Esporas 22-29 x 11-14 nm. Parásito sobre Lecanora rapicola.

Rhizocarpon geographicum (L.) DC

Epitecio pardo verdoso, $\mathrm{K}+$ púrpura.

Cladonia deformis Hoffm.

Podecios escifulíferos, verdeamarillentos, sorediados. En el suelo de cuevas y oquedades, sobre restos vegetales y musgos.

Umbilicaria cinereorufescens (Schaer.) Frey

Talo monofilo, de hasta $4 \mathrm{~cm}$. Cara inferior negra, con láminas perforadas que irradian en torno al ombligo.

Umbilicaria depressa (Ach.) Frey

Talo de hasta $10 \mathrm{~cm}$, monófilo, gris ocráceo. Cara inferior densamente cubierta de ricinas rosadas. En fisuras orientadas al Sur.

Umbilicaria virginis Schaer.

Talo monófilo, 2-4 cm, gris ocráceo. Cara inferior con ricinas rosadas y con pequeñas fosetas. Algunos ejemplares con apotecios de disco poco plisado pueden referirse a la var. meylanii. En paredones orientados al Sur.

Umbilicaria decussata (Vill.) Frey

Talo monófilo, gris muy oscuro, con una retículo de surcos. Cara inferior negra, grisácea hacia los bordes, que presentan perforaciones frecuentes. Esporas 7-11 $14-6 \mathrm{~nm}$.

Umbilicaria nylanderiana (Zahlbr.) H. Magn.

Talo negro mate, de superlicie verrucosa. Cara inferior lisa y negra. En fisuras más o menos nitrificadas.

Acarospora oxytona (Ach.) Massal.

Talo amarillo, orbicular, lobulado, a menudo grande. Lóbulos de superficie áspera. Apotecios planos, de margen persistente. Sobre paredes rocosas no soleadas, a menudo en superticies superverticales y techos, en nuestra zona, orientados al Este y al Sur. Se asocia con líquenes nitrófilos. Las condiciones, aparentemente paradójicas, de disponibilidad de sales, pueden ser debidas a la ausencia de lavado por la lluvia. Por ello este liquen se comporta como ombrófobo.

Aspicilia intermutans (Nyl.) Arn.

Talo gris claro, ligeramente ocráceo. Esporas $20-25 \times 12-15 \mathrm{~nm}$. Talo $\mathrm{K}+$ rojo.

Frecuente. Esta localidad debe de estar próxima a su límite altitudinal. A pesar de su abundancia en los silicatos de toda la España mediterránea, en el momento de redactar este trabajo no pudimos detectar citas de esta especie para Andalucía.

Aspicilia hoffmannii (Ach.) Flag.

Talo gris ocráceo, no raramente verdoso. Esporas globosas, $15-30 \mathrm{~nm}$. Especie eurioica, prefiere los silicatos nitrificados y poco inclinados, pero vive también sobre calizas duras.

Lecanora rupícola (L.) Zahlbr.

Talo blanco grisáceo. Apotecios pruinosos. Esporas 12-15 x 7-8 nm. Frecuente en la localidad, sobre cualquier superficie rocosa, incluso en techos y paredes. 
Lecanora polytropa (Ehrht.) Th. Fr.

Esporas 11-13 x 3,5-4,5 nm. Frecuente, heliófila, pionera en la colonización de superficies expuestas de rocas y piedras, algo nitrófila.

Lecanora achariana A.L. Sm. (L. cartilaginea (Westr.) Ach.)

Lóbulos planos, alargados, más o menos ascendentes. Talo amarillo verdoso. Areolas bordeadas de negro. Ascocarpos numerosos, de disco rosado. Frecuente en convexidades de roca. Bastante nitrófilo.

Rhizoplaca chrysoleuca (Smith) Zopf ( Leccanora rubina (Vill.) Ach.)

Esporas 8-12 x 3-6 nm. Convexidades y cumbres de bloques, en lugares expuestos. Ornitocoprófilo.

Rhizoplaca melanophthalma (Ram.) Leuck. et Poelt

Frecuente. Ecología parecida a la del anterior, aunque se extiende a las paredes verticales.

Rhizoplaca peltata (Ram.) Leuck. et Poelt

Talo casi totalmente cubierto de apotecios grandes, deformes, de disco pardorojizo. Sobre paredes verticales, bien representado en nuestra localidad.

Lecanora gangaleoides Nyl.

Tecio hialino. Esporas 7-10 x 12-14 nm. Es interesante su presencia en un lugar tan elevado.

Candelariella vitellina (Enrht.) Müll. Arg.

Talo formado por granulaciones dispersas, vitelinas. Talo y margen talino $\mathrm{K}+$ rosado. Disperso entre el talo de otros líquenes.

Parmelia scortea Ach.

Ejemplares estériles. Sobre rocas y musgos, en lugares poco soleados, grietas, etc.

Parmelia infumata Nyl.

Talo pardo verdoso, sin apotecios. Isidios al principio cilindricos, después coraloides.

Sobre paredes de roca silícea. En nuestra localidad, invadiendo céspedes de Ramalina prorecta.

Parmelia sorediosa Almb.

Talo verde oliváceo, recubierto de isidios. $\mathrm{K}_{-}, \mathrm{Cl}_{-}, \mathrm{P}_{-}$. Escaso.

Cetraria commixta (Nyl.) Th. Fr.

Lacínias pardo-negruzcas, de borde verrucoso. Esporas de pared gruesa, 7-10 x 4-5 nm. Escaso.

Ramalina obtusata (Arn.) Bitter

Lacínias dilatadas en el borde distal, que es irregular. Superficie surcada y venada. Soralios terminales, en capuchón. En la parte superior de rocas, entre anfractuosidades, en lugares expuestos. Nuevo para la flora hispánica.

Ramalina protecta $\mathrm{H}$. Magn.

Talo verde pálido, de lacínias finamente divididas. Aspecto general pulvinulado, de hasta 3 $\mathrm{cm}$ de diámetro. Soralios más abundantes en la parte terminal de las lacínias. De distribución netamente española, alcanza en nuestra localidad su límite altitudinal. Vive en paredones, al abrigo de irregularidades de la roca.

Alectoria minuscula Nyl.

Talo con aspecto de almohadillas más o menos levantadas del substrato, de color negro brillante. Poco abundante. Segunda cita para la flora española. 
Caloplaca cerina (Ehrt.) Th. Fr.

Esporas $10-15 \times 6-8 \mathrm{~nm}$. Raro en nuestra localidad.

Caloplaca stillicidiorum (Wahlb.) Lynge

Talo epibrio, formado por escamas grisáceas. Apotecios con margen concolor y disco anaranjado. Esporas $12-14 \times 7-8 \mathrm{~nm}$. Sobre musgos y restos vegetales, en grietas y oquedades.

\section{Caloplaca epithallina Lynge}

Talo parásito de otros líquenes (principalmente, de Dimeluena orcina (Ach.) Norm.). Grupos de apotecios de color rojo ferruginoso vivo. Esporas 11-13 x 6-8 nm. Poco citado hasta ahora, y sólo de los Pirineos (Llimona, 1979), donde no parece ser raro a partir de los $2.000 \mathrm{~m}$.

Caloplaca festiva (Ach.) $\mathrm{Zw}$.

Talo grisáceo. Apotecios rojo ferruginoso. Esporas 13-16 x 4-6 nm, más estrechos que en el tipo. Frecuente, a pesar de estar en su límite altitudinal.

Caloplaca anchon-phoeniceon Poelt et Clauz.

Talo poco visible. Grupos de apotecios confluentes, de un bello rojo vermellón. Esporas 10$12 \times 4,6$ nm. Parásito de Dimelaena oreina. Nuevo para España.

Xanthoria elegans (Link) Th. Fr.

En nuestra zona busca lugares más o menos protegidos, sobre todo en paredones orientados al Sur. Nitrófilo. Abundante.

Buellia spuria (Schaer.) Anzi

Talo crustáceo, pequeño, fisurado-areolado, gris parduzco, con hipotalo negro, $\mathrm{K}+$ amarillo. Medula I+ azul. Apotecios incluidos, numerosos. Epitecio N+ rojo. Esporas constrictas, $10-12 \times 6-7 \mathrm{~nm}$. Frecuente

Dimelaena oreina (Ach.) Norm.

Esporas 9-12 x 4-6 nm. Frecuente, sobre roca dura. Algo nitrófilo.

Physcia teretiuscula (Ach.) Lynge

Lóbulos convexos, divergentes, largos. Sobre paredes de cuevas y oquedades, esciófilo y algo nitrófilo.

Physconia muscigena (Ach.) Poelt

Talo gris, pruinoso. Cara inferior negra. Sobre musgos de cuevas y lugares sombreados y más o menos húmedos. Frecuente. 


\section{BIBLIOGRAFIA}

COLMEIRO, M. -1889- Enumeración y revisión de las planlas de la Peninsula Hispano Lusitana e Lslas Baliares. (Liquenes). 5: 473-571. Madrid.

DEGELIUS, G. -1966- Lichens of the summit of the Picacho del Veleta (Sierra Nevada) Spain. A contribution to the flora of high altitudes. Siensk Bot. Tidsk. 60: 338-34l.

ESPINOSA, P. et P. PRIETO -1970- Algunos datos sobre la biometeorología de Sierra Nevada. Ars. Pharm. Granada. 11: 510-516.

HERTEL, H. -1970- Beiträge zur kenntnis der Flechten familie Lecideacia' III. Herzogia 2: $37-62$.

LLIMONA, X. -1979- Líquens; in R. Folch (dir). "El patrimoni natural d'Andorra". Ketres ed. Barcelona. $446 \mathrm{pp}$.

NAVAS, L. -1902- Una excursió científica a la Sierra Nevada. Bulll. Inst. Cal. H. N. any II. 11: 46-50; 12: 68-74; 13: 85-90; 15: 133-199.

POELT, J. -1974- Bestimmungscblïssel Europäischen Flecblen. 757 pp. J. Cramer.

PRIETO, P. et P. ESPINOSA -1971- Ascolíquenes de los Peñones de San Francisco. Sierra Nevada. Cuad. Cion. Biol. Unir. Granada. 1: 95-98.

WERNER, R.G. -1936- Note sur la flore lichénique de l'Espagne méridionale comparée à celle du Maroc. 69 Congr. Soc. Sar. Montpellicr: 179-182.

- 1937. Recherches phytogéographiques comparées sur la flore cryptogamique de l'Espagne méridionale et du Maroc. Bull. Soc. Sit. Nal. Maroc. 17 (1): 32-56.

- 1975. Etude écologique et phytogéographique sur les lichens de l'Espagne méridionale. Reri. Bryol. el Lichénol. 41 (1): 55-83.

- 1979. La flore lichénique de la cordillère bético-rifaine. Collectanea Botanica 11: 401463. 